\title{
LA ALFABETIZACIÓN ECOLÓGICA COMO NUEVA PEDAGOGÍA PARA LA COMPRENSIÓN DE LOS SERES VIVIENTES
}

\author{
José M. Aranda Sánchez ${ }^{1}$
}

Recibido el 13 de julio de 2014, aprobado el 20 de noviembre de 2014 y actualizado el 08 de mayo de 2015

DOI: 10.17151/luaz.2015.41.20

\section{RESUMEN}

El objetivo central de este artículo es analizar las bases, la plataforma y los principios de la pedagogía que Fritjof Capra ha denominado Alfabetización Ecológica, para referirse a u na particular forma de ab ordar la educación ambiental, ya no desde el salón de clases sino a partir del lugar mismo donde se busca intervenir, teniendo como soporte filos ófico-científico a la Ecología Profunda; y co $\mathrm{n}$ una va sta experiencia de trab ajo colectivo que ha rebasado fronteras geográficas, y no exige má s doctrina que la voluntad y humildad para seguir los pasos que permitan descubrir los problemas y actuar en consecuencia, en el aquí y ahora de nuestras complejas relaciones comunitarias con los sistemas vivientes.

\section{PALABRAS CLAVE}

Educación ambiental, alfabetización ecológica, ecología profunda, ecosofías, sistemas vivos.

\section{ECOLOGICAL LITERACY AS A NEW PEDAGOGY FOR THE UNDERSTANDING OF LIVING THINGS.}

\begin{abstract}
The central objective of this ar ticle is to analyze the bases, the platform and the prin ciples of the $p$ edagogy Fritjof Capra ha $s$ called Ecological Literacy to refer to a particular way of addressing environmental education, no long er from the classroom but from the very place where it seeks to be intervened having Deep Ecology as the philosophical-scientific support and with a vast experience of collective work that has gone beyond geographical borders, and does not require more doctrine that willingness and humbelness to follow the steps to discover the problems and act accordingly in the here and now of the complex community relations with living systems.
\end{abstract}

\section{KEY WORDS}

Environmental education, ecological literacy, deep ecology, ecosophy, living things. 


\section{INTRODUCCIÓN}

Sin duda la creciente preocupación por las cuestiones ecológicas y los cambios curriculares del sistema educativo han favorecido la progresiva entrada de los temas ambientales en la escuela. No obstante, por lo gene ral esos temas son tratados de mane ra superficial, es decir, sin ir más allá de los enunciados generales, "por encima" de los problemas, y sin que logre promover en los estudiantes la comp rensión y explicació $\mathrm{n}$ de lo $\mathrm{s}$ procesos ambientales como sistemas complejos ${ }^{2}$ (Martínez, 1993; Harvey y Reed, 1997; Eve et al., 1997; Martíne z y Gocho, 1998; Hayles, 2003; Bocara, 2004; García, R., 2005; Reynoso, 2009).

Asistimos a un momento en que tanto niños como jóvenes y adultos queremos hacer algo por cuidar el planeta; pero la información disponible es en muchos casos insuficiente y, otras veces, confusa y co ntradictoria. Así, es frecuente que en la s escuelas se lleven a cabo campañas "ecológicas"; sin embargo, estas acciones por lo general quedan descontextualizadas de la propuesta pedagógica que debiera sustentar la Educación Ambiental (Damian y Monteleone, 2002).

Lo anterior se debe a que los do centes se encuentran ante el dilema de incorporar los temas que la sociedad demanda; pero que no e stuvieron presentes en su forma ción académicaprofesional. Por lo mismo, no cuentan con los marcos didácticodisciplinares ni las experiencias formativas previas. Entonce $s$ tienen que enfrentarse al desafío de en señar lo que no aprendieron, logrando exponer los temas sin que esa enseñanza pueda incidir en un a formación para la su stentabilidad como tendría que ser (Jonassen y Land, 2000).

Tal es el caso de una práctica do cente muy re currente en las escuelas con la p opular campaña de recolección del atas, botellas de plástico o de reciclado de papel. Por lo regular, se convierte en una moda y se diluye el objetivo. De ahí que, en lugar de apuntar a la modificación de determi nados hábitos consumistas y apoyar I a toma d e conciencia acerca de I a innecesaria cantidad de basura que se produce y el impacto que tiene en el ambiente, el propósito de la campaña se reduce a juntar un cierto volumen de material reciclable a fin de canjearlo por algún premio para la escuela (una computadora o una videocasetera). El punto es que "juntar latitas" y otras acciones pasa a ser un fin en sí mismo qu e no siempre es resignificado a través de una práctica escolar contextualizada por un antes y un después (cambio de actitud), ni adecuadamente encuadrado en una secuencia didáctica que permita conceptualizar, reflexionar y alcanzar una mirada crítica en to rno a nuestras decisiones y acciones cotidianas, así como evaluar el imp acto que e stas tienen en el ambiente (Damian y Monteleone, 2002).

A pesar de lo anterio $r$, múltiples y diversos son los intentos y experiencias institucionales relacionadas con la Educación Ambiental contextualizadas en la realidad local donde se abren a la comunidad y que no se limitan a I a escuela. Especialmente, suelen ser exitosas las experiencias que logran un trabajo 
interdisciplinario, por ejemplo, entre las áreas de ciencias naturales y ciencias sociales, donde se integran organizaciones sociales (Gutiérrez, 1995; Jonassen y Land, 2000; Aguilar y Maihol'd, 2001; Caride y Metra, 2001; Wenger, 2001; West, 2002; Sureda y Colom, 2003; Capra, 2003; Carabias et al., 2009).

En este a rtículo se expone una pedagogía diferente a I a dominante en las prácticas convencionales, que está vigente y generando múltiples experie ncias ecológicas en diferentes ambientes y países, y que interesa considerar en sus fundamentos y plataforma met odológica, resaltando la importancia de re-plantear la cuestión desde la ecología profunda, y resituando el problema de la práctica ecológica en el momento mismo de la planeación educativa, a partir de la toma de conciencia de que, e $\mathrm{n}$ estricto sentido, la mayor parte d e nosotros somos unos analfabetas ecológicos, puesto que apenas tenemos unas cuantas nociones generales y superficiales de los complejos entretejidos que forman y confo rman la vida en el planeta, y que tal vez por ello aún no asumimos con decisión y conocimiento los compromisos que las crecientes crisis ecológicas exigen.

El escrito se integra en tres apartados: una presentación de la ecología profunda de Arne Naess; la nueva perspectiva de los sistemas vivos de Fritjof Capra, y principios de la alfabetización ecológica, terminando con algunas reflexiones derivadas del análisis y una prospectiva que busca ser a la vez in vitación a repensar y actuar en consecuencia.

\section{BASES DE LA ECOLOGÍA PROFUNDA}

Si bien desd e mediados de los n oventa Fritjof Capra (1999) afirmaba que la Ecolo gía Profunda constituía un $\mathrm{n}$ uevo paradigma, eminentemente holístico, a partir de una visión integrada del mundo, y no como una serie discontinua de partes, actualmente esa perspectiva no solo sigue vigente sino que además ha adquirido mayor relevancia, en la medida en que no disocia a los seres humanos, ni o bjeto $u$ artefa cto alguno, d el entorno natural. Es decir, concibe y percibe al mundo no como una colección de objeto s separados entre sí, sino como una compleja red de procesos interconectados e interdependientes que configuran una trama, de la cual los seres vi vos son una fibra.

En su exp resión más abierta, la ecología profunda se presenta más como un movim iento que una filosofía. Conde nsa importantes conjuntos de personas de diferentes formaciones y ocupaciones, que coinciden y promueven el mismo estilo de vida y llevan al cabo campañas y acciones colectivas en defensa del ambiente, además de partidos verdes que participan en el terreno político de varios países, incluso un grupo ultra que toma acciones ante problemas críticos de la biósfera (Naess, 1988). 
De hecho, es un movimiento radical en dos aspectos: primero, debido a que busca plantear un cuestionamiento "profundo" acerca de I as prioridades valorativas y de lo $\mathrm{s}$ supuestos filosóficos (tanto ontológicos como filo sóficos y ético s) que se enlazan en nuestra forma de vivir y de pensar; segundo, y como consecuencia de lo anterior, puesto que promueve un cambio de fondo de las sociedades humanas que deje ya la idea del crecimiento económico como lo principal, en aras de una vida "simple en los medios pero rica en lo s fines". Sob ra decir que bajo esta premisa, al movimiento se le ha tachado de ingenuidad, sobre todo por desconocer los intereses políticos y el afán de lucro que se ha $n$ impuesto en nue stras sociedades "desarrolladas".

El sentido de la profundidad que proclama el movimiento apunta a señalar que es una reflexión en torno a los fundamentos de la organización social, así que, para quienes cuestionan el modelo de desarrollo y los est ilos de vida imperantes, no existe justificación alguna que dé cuenta de que I a sociedad, la educación, e incluso la religión, resultan beneficiadas para toda la vida en el planeta con siderado globalmente. Por ello es que, a continuación se pregunta qué se requiere para lograr los cambios necesarios. Más aún, no se limita la crítica a un enfoque científico: se tiene la obli gación de una visión total. Por ello es que, a pesar de que la mayoría de los seguid ores no son filósofos, ese "cue stionarlo todo" los hace conscientes de su propia filosofía (o ecosofía), como nombra Naess a ese conjunto de "visiones totales" que surgen en parte de la ecología (Issa , 2003).

Tal comol o hiciera Sócrates, Naess busca producir un cuestionamiento hasta que los demás tengan el saber en torno a su posición sobre las cuestiones básicas de la vida y la muerte (Naess, 1969; Merchant, 1992; Sessions, 1995).

Ahora bien, esas "ecosofías" pueden presentar muchas diferencias (hasta llegar a la oposición) entre los partidarios de la ecología profunda; pero lo principal es la motivación de todos en cuanto a su vinculación especial con la naturaleza, en la medida en que procede de sus creencias y actitudes más afianzadas: es la palanca y soporte para garantizar la continuidad y eficacia del movimiento (García, M., 2005).

Sin embargo, la otra orientación que perfiló Naess para insistir en la idea de ecología "profunda", destaca la diferenciación entre esta última perspectiva y aquella considerada como superficial, que si bien también muestra preocupación por el planeta, no deja de estar anclada a una idea antropocéntrica de los problemas ecológicos con base en la que la naturaleza tiene que protegerse básicamente por el val or instrumental que tien e para los humanos. Es ahí donde la ecología profunda critica duramente la desviación socialmente injusta a e scala mundial, implícita en la ideología que nutre al movimiento de "reforma superficial". En otras palabras, no basta con el combate a la contaminación y a la explotación desmedida de recursos, ya que el propósito es principalmente la salud y opule ncia de los habitantes de países 
desarrollados. Es entonces cuando el movimiento se radi caliza y llama a una revaloración metafísica del significado de ser humanos. De ninguna manera se trata de repensar el lugar que le corresponde a los hombres en la totalidad del cosmos, sino de cambiar la perspectiva de que cada persona en el planeta se maneja y decide desde "su verdadero yo" (Issa, 2003).

En estricto sentido, el núcleo filosófico de la ecología profunda se encuentra constituido por dos postulados. El p rimero es la afirmación de un holismo de carácter místico a partir del cual los individuos únicamente existen como tales relativamente, o más bien, solo pueden ser co ncebidos como tales en virtud de sus relaciones con los demás seres vivientes. La vida no es más que una red dentro de la cu al los se res individuales constituyen nodos o ce ntros de inte racción espaciales. Sin embargo, no existe un ser vivo al margen del contin uo biológico, y este es en realidad un sistem a de rela ciones completamente integrado, quiere decir, una totali dad indiferenciada que se encuentra en flujo constante. La idea-fuerza para plantear e sta ontología hiperholista la obtiene de Mahatma Gandhi, quien asimismo se inspiró en la escuela vedanta advaita de filosofía hin duista, que sostiene la tesis de la unidad esencial de todo lo existente.

De este primer supuesto, a saber, que no hay un auténtico argumento para diferenciar entre yo y no-yo, entonces, y este es el segundo postulado de la ecología profunda, quien se identifica con la totali dad del universo no únicamente tiene la vi sión (ontológica) recta de la s cosas, sino que además ha alcanzado un grado de madurez psicológica que deberá traducirse necesariamente en una transformación del trato que le dispensa la naturaleza (Naess, 1995).

En efecto, la regla de la Autorrealización exige un llamado a que los hombres expandan la conciencia que tienen de sí mismos, y con ello dejen atrás la i dea común y egoísta de que somo $\mathrm{s}$ individuos separados con intereses particulares, para orientarnos correctamente hasta alcanzar un sentimiento de i dentificación con el to do. Entonces, la plena realización, así comprendida, consiste en una verdadera trascendencia3 hacia un estadio religioso. Para Naess esta perspectiva conlleva, entre otras cosas, la presencia de un significativo impulso motivacional para la conservación ecológica (Passmore, 1978).

De tales argumentos se desprenden dos co nsecuencias de la mayor relevancia. Ante todo, el estilo de vida de a quellos que han experimentado el cambio de su ego restringido en el ego ecológico no puede seguir orientado -como sucede en el capitalismo contemporáneo- por el deseo de mayor nivel de consumo. El cambio radical que ha sufrido su visión del mundo lo impulsa necesariamente a buscar una vida más sencilla; aunque también más llena de experiencias, aceptando una disposición de medios requeridos para disfrutarla. Naess insiste mucho en no dejar de revisar nuestros valores habituales a favor de valo res que enaltezcan la experiencia en todos sus aspectos. 
La otra consecuencia es de mayor peso filosófico, en la medida en que ese cambio en la percepción del mundo es la base para poder llevar a cabo la acción acertada. Esto es, que se da un decisivo apartamiento de la ética (incluso la ambiental) y de la moral y el deber, para aproximarse a la ontología, como soporte para la a cción correcta. Este cue stionamiento llega incluso a poner en duda el fun damento de la ética para la conservación. Esto es, si la gente percibe el cuidado que le brinda al a naturaleza como un sacrificio de su interés egoísta, la ética que se lo demanda como obligación no conseguirá eficacia práctica. El mejor camino es la identificación del individuo con el universo natural, ya que será entonces cuando la conservación se aprecie en términos de un inte rés propio, auténtico, y la acción se realizará por amor y con gusto, mas no por deber que se considera exigido desde afuera (Naess, 2002).

\section{LA NUEVA PERSPECTIVA DE LOS SISTEMAS VIVOS}

Mucho antes de pro poner algunos principios, relativamente sencillos y claros, con base en los cuales impulsó una nueva pedagogía ecológica, Fritjof Capra lle vó a cabo un profundo estudio, análisis y reflexión en torno al problema ecológico y los sistemas vivientes, que lo llevó no solo a consultar y deli berar con los pri ncipales científicos de todo el mundo durante mediados y finales de los años ochenta, sino también a explorar sobre un posible "nuevo" paradigma en la enseñanza ambiental, producto precisamente de ese ir más allá en la explicación de los problemas ecológicos urgentes de enfrentar; pero además, acerca de otro camino para asumir individual y colectivamente la responsabilidad social que nos corresponde.

La obra central de Capra, y de la cual haremos una narraciónresumen parcial, con el propósito de ir llevando al I ector por la lógica que el autor encontró más idónea en su afán de presentar en un todo coherente la síntesis de su perspectiva, es La trama de la vida, ampliamente reconocida por expertos, filósofos y estudiosos de los temas ambientales como un aporte que marcó un momento de ruptura y re-comienzo de la reflexión profunda de nuestro lugar y compro miso como seres humanos ante la complejidad de la vida y su so stenibilidad ahora y en adelante (Capra, 1999).

Se trata de un recorrido que nos va mostrando, a veces a pasos y en mome ntos a grandes saltos, cómo el e sclarecimiento del problema ecológico no fue tarea fácil ni exenta de controversias, principalmente científicas, que de rivaron en una dif erente forma de comprender la crisis ambiental y, en consecuencia, de ofrecer una alternativa para asumir nuestra responsabilidad, una vez que hemos logrado abordar la cuestión desde la ex igencia del pensamiento, es de cir, cuando hemos avanzado desde la apariencia y la contemplación hasta la comprensión y la acción consecuente (Arendt, 2002). 
El primer pasaje fue mostrar cómo, desde la física, vale decir, de la ciencia exacta por excelencia, se registró un cu estionamiento de fondo en torno al viejo paradigma que concebía al universo como un sistema mecánico compuesto de piezas, al cuerpo como una máquina, la vida social como un campo de lucha por la supervivencia y el poder; la creencia en el progreso material sin límites a través del crecimiento económico y te cnológico, así como la idea de que el patriarcado y la consiguiente dominación sobre las mujeres por parte de los ho mbres era algo así como una 'ley natural'.

El nuevo paradigma, que ya hemos analizado suficientemente en el punto anterior, de la Ecología Profunda es sin duda el eje y pivote del replanteamiento, por lo que no agregaremos nada más.

El paso siguiente consistió en considerar la e mergencia del pensamiento sistémico como un segundo momento en el cual la humanidad avanzó para esclarecer y "resolver" un viejo dile ma filosófico y científico: la relación entre el todo y las partes que lo componen. En efecto, para Capra la secuen cia llevó al conocimiento científico del estudio de las partes a la comprensión del todo, en la medida en que se avanzó desde el paradigma mecanicista al ecológico, que supone lo holístico u organicista.

No se trató de un cambio lineal ni $\mathrm{m}$ ucho menos, sino de u $\mathrm{n}$ 'péndulo caótico', a partir de la i rrupción del pensamiento sistémico, encabezado por los biólogos, que antepusieron la percepción de los organismos vivos como totalidades integradas. Más adelante, la p sicología de la Gestalt ${ }^{4}$ y la nu eva ecología habrían de esclarecer el esquema y darle proyección hacia otras áreas del conocimiento.

Para Capra, la tensión entre mecanicismo y organicismo tiene mucha historia, y se refiere a la añeja oposición entre substancia (materia, estructura, forma, cantidad) y forma ( $p$ atrón, orden, cualidad). Así, ante el mecanicismo de Descartes el primer cuestionamiento radical, si bien surgió en el arte, la literatura y la filosofía del XVIII, fue en el XIX cuando Immanuel Kant opuso las máquinas a los organismos, otorgándoles a estos la capacidad de autoproducción y autoorganización (Capra, 1999).

Desde el mecanicismo del siglo XIX, reforzad o por los descubrimientos en biología, especialmente en microbiología, se avanzó a través de las controversias con el vitalismo, hasta llegar a la biol ogía organicista, que en oposición al mecanicismo y al vitalismo, tomó el probl ema de la forma biológi ca, sembrando la semilla de lo que hoy denominamos pensamiento sistémico. En esa vía, se exploró el concepto de organización, que i ría reemplazando la antigua noci ón de función en fisio logía, lo que propició un cambio del pensamiento mecanicista al sistémico. De ahí se dio un importante paso pa ra identificar una de la $\mathrm{s}$ propiedades especiales de to da manifestación de vida: la tendencia a construir estructuras multinivel de sistemas dentro de sistemas. Donde cada uno de ellos forma un todo con respecto a sus partes, siendo a la vez parte de un todo mayor. De e sta 
manera, las células se combinan para formar tejidos, estos para conformar órganos y estos a su vez $\mathrm{p}$ ara configurar organismos (Capra, 1999).

Estas ideas de la biología organicista, por lo tanto, suponían una concepción sistémica de la vida e $\mathrm{n}$ términos de conectividad, relaciones y contexto. Según e sta visión, las propie dades esenciales de un orga nismo o sistema viviente, son cuali dades del todo que ninguna de las partes presenta. O sea, emergen de las interacciones y relaciones entre las partes.

Como consecuencia de estos cambios epistemológicos, Capra afirma que resultó en un shock para la cien cia del siglo XX ya que implicó la constatación de que los sistemas no pueden ser comprendidos por $\mathrm{m}$ edio del análi sis, es decir, que las propiedades de la $s$ partes no son intrínsecas, sino que únicamente pueden ser comp rendidas en el contexto de un conjunto mayor. Lo que deriva en una inversión de los términos: las propiedades de las partes solo pueden conocerse desde la organización del conjunto. De ahí que el pensamiento sistémico no se concentre en los componentes básicos, sino en los principios esenciales de organización.

En tanto que los biólogos organicistas tenían ante sí la totalidad irreductible en los organismos, los físicos cuánticos en los fenómenos atómicos y los $p$ sicólogos de l a Gestalt en I a percepción, los e cólogos la encont raban en sus estudios de comunidades de plantas y animales ${ }^{5}$. Y así fue com o el ecólogo botánico británico A.G. Tansley refutó la noción de superorganismo y propuso el término "ecosistema" para describir a las comunidades de animales y plantas. Actualmente se entiende por ecosistema una comuni dad de organismos y su entorno físico, intera ctuando como una unidad ecológica, indicando la aproximación sistémica de la ecología.

Para Capra, la nueva ciencia de la ecología (Deléage, 1993) enriqueció el emergente pensamiento sistémico con I a introducción de dos nuevos conceptos: comunidad y red. En una palabra: la mayoría de los organismos no solo son miembros de comunidades ecológicas, sino además complejos ecosistemas que contienen cantidad de organismos más pequeños dotados de relativa auto nomía; pero integrados armoniosamente en un todo funcional. Por ello es que la "trama de la vida" es una expresión, con mucha historia, para dar cuenta del entretejido e i nterdependencia de todos los fenómenos; donde los sistemas vivos son rede $s$ en interacción con ot ros sistemas (Jacob, 1994; Penfield, 1995; M onod, 1998; Wagensberg, 2001; Rozsak, 2003; Diamond, 2007; Schödinger, 2012).

Lo principal, entonces, radicó en el cambio de las partes al todo o bien de objetos a relaciones. Puede decirse que se trató de un cambio de e squemas, de diagramas, por eso es que pa ra el pensar sistémico las relaciones son prioritarias. Sin embargo, la red de relaciones se describe en términos de su correspondiente red de conceptos y mo delos, ninguno de lo s cuales es más 
importante que otro. Asim ismo, el viejo paradigma de la certeza del conocimiento científico se ve cuestionado por el nuevo paradigma que acepta que todos los conceptos y teorías científicas son limitados y aproximados: la ciencia nunca puede ofrecer una comprensión completa y definitiva (Bateson, 1998).

Esto último conlleva al pensamiento en términos de procesos, es decir, que en la ciencia sistémica cada estructura es vista como expresión de procesos subyacentes. En esta lógica, el metabolismo de la célula viva, que combina orden y actividad de un modo que no puede ser descrito por la ciencia mecanicista, implica miles de reacciones químicas, que se producen simultáneamente para transformar los nutrientes de la célula, sintetizar sus estru cturas básicas y eliminar lo $\mathrm{s}$ productos de desecho. "El metabolismo es una actividad continua, compleja y altamente organizada" (Capra, 1999, p. 63).

Más adelante, debido a los esfuerzos de Bertalanffy, el pensamiento sistémico era ya un movimiento $c$ ientífico de importancia; y él mismo in sistía en q ue el pensamiento evolucionista, es de cir, en términos de cambio, crecimiento y desarrollo, requería una nueva ciencia de la complejidad. Y si bien se reconoce a la termodinámica y su "segunda ley" como expresión pionera de la nueva aproximación, o sea, el trascendente principio de la disip ación de la e nergía, o mejo $r$ conocido como entropía, apenas se introducían los principios de irreversibilidad, de un vector de tiempo en la ciencia de la época.

Por ello, uno de los mé ritos de Bertalanffy fue haber acuñado el término "equilibrio fluyente" para dar cuenta del funcionamiento de los sistemas abiertos, que a diferencia de I os sistemas cerrados -característicos del as máquinas-, describían el equilibrio dinámico de los sistemas vivientes, razón por la cual se imponía una "nueva termodinámica" donde la autorregulación de las estructuras disipativas es la regla y princi pio cibernético (Bertalanffy, 1974).

De ahí a la autopoiesis, es decir: la organización de lo vivo, Capra muestra con abundantes referencias la conexión exacta entre autoorganización y vida, enf atizando el proceso de cognición, en una nueva comprensión, mostrando cómo se trata de una red de procesos de producción, en la cual la función de cada componente es participar en la producción o transformación de otros componentes de la red (Capra, 1999).

Este paso fue crucial y de rivó en la concepción de Gaia, la tierra viva, en una con cepción que, haciendo eco a las reflexiones de Lovelock, avanzó junto con Lynn Margulis hasta develar gradualmente una compleja red de bucles de retroalimentación que, afirmaron, era la responsable de la autorregulación del planeta (Lovelock y Margulis, 1974).

Así, la mezcla de sistemas vivos, como los animales, y no vivos como rocas o el agua, conformamos un todo orgánico con base en la complejidad del sistema. 
Esa visión de los seres vivos como redes autoorganizadoras, cuyos componentes se encuentran interconectados y son interdependientes, nos dice Capra, tiene referencias en la historia de la filosofía de las ciencias; pero las herramientas matemáticas para estudiar la intercon ectividad no-lineal, típica de las redes, solo se dio con las llamadas matemáticas de la complejidad, o también teoría de los sistemas dinámicos, que es precisamente una teoría matemática, y no una teoría de los fenómenos físicos (Jantsch, 1980; Guattari, 1996; G uiddens, 1998; Capra, 1999; Maturana y Varela, 2000, 2001; Prigogine y Stengers, 2004).

Específicamente, estas matemáticas incluyen: una referencia a la ciencia clásica y el álgeb ra superior; las Ecuaciones diferenciales, la retroalim entación y las itera ciones. Las huellas del caos en Poincaré, las trayectorias de los espacios abstractos, los atractores extraños, la ge ometría fractal, I os números complejos, y los patrones dentro de patrones. De tal manera, que la nueva síntesis permite confirmar la estructura de los sistemas vivos, a saber: la $s$ estructuras disipativas, directamente relacionadas con I os cambios; solo que a hora se trata d e estructuras que se encuentran en no-equilibrio y no-linialidad. Lo que además se vin cula con lo que Prigogine (2008) establece como irreversibilidad e indeterminación de los procesos.

Entonces, la relación entre orden y d esorden queda removida, toda vez que del desorden puede surgir el orden; e incluso del orden al desorden que muestra la idea del movimiento de estado improbable a estado probable, condición que mate máticamente puede definirse como el estad o atractor de equilibrio térmico (Capra, 1999).

Con todas esas nuevas bases, y la convicción de que en los seres vivos existen bucles catalíticos ${ }^{6}$ (quiere decir, procesos químicos no-lineales, irreversibles) que conducen a inestabilidades a través de la reiterada retroalimentación autoamplificadora, y sigu iendo las reflexiones de Prigogine, Capra nos conmina a integrarnos más plenamente con la naturaleza, a la cual le debemos respeto, cooperación y el diálogo, un nuevo diálogo con las entidades no-humanas.

Y si bien el desarrollo de su planteamiento entorno a I a autopoiesis $^{7}$, desde nuestro punto de vi sta, queda insuficientemente formulada, no deja de ser interesante pensar en la fusión de los mundos simbólico y natural, j ustamente a partir del le nguaje humano. Lo que nos remite de nuevo al sistema Gaia y a los ecosistemas, como las unidades básicas de los sistemas vivientes, en su articulación con las entidades no bióticas. Se trata de comprender y visualizar al universo en su totalidad, donde la vida existente y los ecosistemas operan en forma de redes, a partir de componentes que interactúan entre sí de tal mane ra que la re d entera se autorregula, organiza y funciona con su ento rno. Así, el despliegu e de l a vida no s conduce a saber que sabemos, es decir, al autoconocimiento, característico de la psique humana. 


\section{PRINCIPIOS DE UNA PEDAGOGÍA PARA LA ALFABETIZACIÓN ECOLÓGICA}

Del anterior recorrido, Capra nos lleva finalmente a reflexionar en el hecho de que somos unos verdaderos analfabetas ecológicos; pero que podemos avanzar para lograr un verdadero conocimiento ecológico el cual, de sde su pe rspectiva, tendría que partir de los siguientes principios: 1) interdependencia entre todos los integrantes de una comunidad ecológica, ya que se encuentran interconectados en u na vasta y com pleja red de relaciones, la trama de la vida; 2) el carácter cíclico de lo s procesos ecológicos, puesto que los circuitos de retroalimentación son caminos a través de los cuales los nutrientes son continuamente reciclados. Entender que comunidades enteras de organismos han evolu cionado de este modo a lol argo de miles de mill ones de años, usando y reciclando sin cesar las mismas moléculas de minerales, agua y aire; 3) la asociación es otro de los rasgos esenciales de las comunidades sostenibles, donde los intercambios cíclicos de energía y re cursos en un ecosistema se sostie nen en una cooperación omnipresente, o se a, al establecer vínculos, vivir incluso unos dentro de o tros y coo perar. Se trata de aspect os distintos de un mismo patrón de organización, para lograr la máxima sostenibilidad ${ }^{8}$.

De los anteriores principios, Capra desprende dos nu evas características a tomarse muy en cuenta en los sistemas vivos: la flexibilidad y la diversidad. En el primer ca so, sugiere además una correspondiente estrategia de resolución de conflictos, ante las inevitables discrepancias en to da comunidad ecológica; mientras que el papel de la diversidad se encuentra íntimamente ligado a su estructura en red. Así, en los eco sistemas, la complejidad de su red es consecuencia de su bio diversidad, por lo cual e sa comunidad será e cológicamente resistente. De ahí que para el autor, la su pervivencia de la humanidad dependerá en gran medida de nuestra capacidad de comprender los principios de ecología y vivir en consecuencia, es d ecir, dependerá de nuestra alfabetización ecológica (Pomier, 2002).

Ahora bien, sobre la base de los anteriores principios, se requiere emprender de otra mane ra la tarea de educación ecológica, cambiando significativamente el esq uema y el pro ceso de alfabetización ecológica, lo que su pone, además, considerar lo siguiente (Capra y Luisi, 2014):

1) Replantear el currículum. Este prin cipio establece que e $s$ necesario desacralizar el currículum entendido como conjunto de temas y lecciones predeterminadas, listas para su aplicación en los educandos, para impulsar una concepción diferente, donde no cabe la idea de un cu rrículum de "talla única", si no que este existirá en todo lugar donde se g enere aprendizaje. Bajo e sta perspectiva, se buscará que cada quien y siempre en colectivo establezca los contenidos que su propia motivación y experiencias personales les lleven. Así, los $\mathrm{n}$ iños pueden encontrar relevancia y significado en el aprendizaje cuando ellos están metidos hasta las rodillas en un río o midiendo los efectos 
de la restauración de los pájaros cantores y los sauces. Cuando las comunidades-escuelas están profundamente comprometidas en resolver problemas de restauración, ellos practican una capacidad esencial para la soste nibilidad; pero penosamente faltante en muchos currículums: la capacidad para la compasión, extendiendo la vigilancia a todas las formas de vida.

2) Partir de la teoría de los sistemas vivientes. La teoría de los sistemas funciona adecuadamente como un marco científico que requiere un cambio de percepción hacia una nueva manera de pensar basada en las rel aciones, el contexto, y la s conexiones. La pregunta es: ¿Existe un patrón de organización común que pueda ser id entificado en todos I os seres vivos? Así es, y su principal propiedad es que se trata de un patrón en forma de red. Por ello, donde quiera que encontremos sistemas vivos organismos, partes d e organismos o co munidades de organismos-, podremos observar que sus componentes están dispuestos en forma de red. Si vemos la vida, vemos redes. Asimismo, ha quedado en claro que los seres vivos comparten la emergencia espontánea de ord en, conocida científicamente como "autoorganización". Y este proceso incluye la creación de nuevas estructuras y nu evos modelos de co mportamiento, es decir, procesos de desarrollo, aprendizaje y evolución. Asimismo, la autoorganización se da únicamente cuando el sistema se encuentra alejado del equilibrio. Finalmente, implica la interconectividad no-lineal de los componentes del sistema.

3) Considerar la sabiduría de los pueblos originarios. Esta "nueva manera" de pensar, de percibir el mundo en términos del contexto y las conexiones, es tam bién sabiduría ancestral. Los pueblos indígenas se han sostenido ellos mismos a través del tiempo en comunidades entendidas para incluir a los humanos, a las otras criaturas vivientes, y a la Tie rra. Es importante asimilar cómo las maneras de educar que estuvieron una ve $z$ indisolublemente integradas en la vida de una comu nidad deben ser ahora conscientemente refortificadas, en su propio lugar, y hasta en un mayor grado en las sociedades industrializadas y altamente móviles. En las comu nidades sostenibles, el compromiso de la comunidad total es ponderado como esencial para el bienestar de todos.

4) Una pedagogía basada en el lugar (situada). Los niñ os necesitan comprometerse organizadamente con los si stemas vivientes y con la vida $d$ e las personas quienes viven gracias a estos sistemas. Antes de que los estudiantes sean introducidos a un conocimiento disciplinario más avanzado, ellos deberían ser inmersos en hábitats y comu nidades tal como e stos ocurren, fuera de los límites construidos en los salones de clases -como los ríos, las montañas, los conucos, los humedales, los jardines, los bosques, los lagos, las islas-. De tal compromiso crece la reverencia que es capaz de transformar el aprendizaje del simple conocimiento a u na pasión por preservar e sos lugares. La enseñanza situada, al establecer un vínculo entre la educación y la vida, permite generar prácticas educativas auténticas. Se trata de un ap rendizaje comprendido como proceso multidimensional de apropiación cultural, ya que se I ogra una experiencia que incluye el pensamiento, la afectividad y la acción. 
5) Educación basada en proyectos. Las perspectivas experiencial y situada pla ntean el problema de la organización y secuencia de los contenidos de la enseñanza o de la estructura del currículo en términos de saberes y h abilidades $\mathrm{o}$ competencias que la p ersona debe lograr para afrontar los problemas, necesidades y asuntos relevantes que se le plantean en los entornos académicos y so ciales donde se desenvuelve. De esta manera, las experiencias educativas en que participan los estudiantes en forma de actividades propositivas y auténticas, organizadas en forma de proyectos, constituyen los elementos organizadores del currículo. Este debe ofre cer al alumno situaciones que lo conduzcan a un crecimiento continuo, gracias a la interacción entre la $\mathrm{s}$ condiciones objetivas o sociales e internas o personales, es decir, entre el entorno físico y social y las necesidades, intereses, experiencias y conocimientos previos de los alumnos.

6) Conformación de comunidades de aprendizaje. Esta es la profunda lección que necesitamos aprender de la naturaleza. La manera de sostener la vida e s construir y mantener la comunidad. La comunidad es esencial para entender la sostenibilidad, y también es esencial para enseñar la ecología de la manera multidisciplinaria que requiere. En las escuelas, varias disciplinas necesitan ser integradas para crear un currículum orientado ecológicamente. Obviamente esto solo es po sible si profesores de varias disciplinas colaboran, y si la administración de la escu ela hace que tal colabo ración sea posible. En el proyecto se establ ecerá la conformación de comunidades de aprendizaje en la medida en que diversos grupos de estudiantes y docentes participen en la integ ración de redes donde se generen las experiencias educativas situadas, con ba se en proyectos, $y$, como $p$ rioridad, que re sulten en un verdadero servicio a la comunidad.

7) Traduciendo teoría en práctica. La perspectiva experiencial, de "aprender haciendo" y el pensamiento reflexivo, en el caso de la Educación Ambiental adquiere especial relevancia, por lo que esta propuesta plantea una secu encia pedagógica para experimentar con el aprendizaje, en los siguientes términos: a) En primer lugar se buscará que los estudiantes descubran un problema, vale deci r, se den cu enta de cómo funcionan los sistemas vivientes, de su relación con el medio abiótico, de tal manera que, b) Exploren ese medio, profundicen en los factores que intervienen, encuentren las causas y ava ncen a la comprensión de lo sucedido, promoviendo que se motiven a c) Enseñar a otros niños y do centes su descubrimiento $y$ entendimiento del problema, lo que conducirá su esfuerzo a tratar de d) Cambiar esas condiciones, encontrar alternativas viables, para lo cual tendrán que, e) Actuar, entrelazando su hallazgo con otras historias, es decir, estableciendo vínculos con la comunidad de la que forman parte.

8) Asumir la Responsabilidad personal. Por la vida en gen eral y por los sistemas vivientes de su entorno en particular. Lo más importante es tomar conciencia de la necesidad de impul sar formas y mo delos sostenibles de vida, difundirlos y actuar en consecuencia, ya no como una tarea escolar o una acción 
aislada, sino en términos de un proyecto de vida comprom etido con la sostenibilidad. Lo que implica considerar que la Educación Ambiental no termina jamás, y que la responsabilidad social debe asumirse como un com promiso ético que se tiene con la sociedad en su conjunto y con el pl aneta en su complejidad creciente.

9) La alfabetización ecológica es una forma de cultivar la inteligencia emocional, social y ecológica, a fin de ge nerar conocimientos, habilidades y valo res esenciales para una vida sostenible. Por ello, in cluye: Descubrir, explorar, enseñar, cambiar y compartir. Como la secuencia a seguir en la aplicación de esta pedagogía, ya que se trata de una serie de experienciasacciones por medio de las cuales, básicamente los niños (y prejóvenes), accederán a esta diferente forma de conexión con la naturaleza. Veamos brevemente cada uno de los momentos en sus rasgos principales:

a) Descubrir. El conta cto directo con la naturaleza tiene como propósito encontrar relaciones que I a propia trama de la vida ofrece; aunque puedan estar encubiertas o no ser visible $s$ a primera vista; pero que es posible de-velar a partir de la vivencia inmediata. Se trata de iniciar el proceso educativo no con teorías - conceptos, sino con la operación racional-afectiva de la indagación, lo que supone una búsqueda inicial para dar con los factores de la situación observada. $Y$ ese transparentar los hechos supone no ú nicamente el resultado de un impulso investigativo, que existe en todos los human os y otros seres vivos, sino a demás el establecimiento de un vínculo entre quien descubre y eso "real" de su descubrimiento; pero que a la ve z tiene otras dos dimensiones, a saber: lo simbólico y lo imaginario. El primero para dar cuenta de los conceptos con los cuales accedemos a la naturaleza; mientras que lo imaginario expresa justamente el peso de la imagen en cuestión, de tal manera que los tres registros: real, simbólico e imaginario, entran en acción en el mom ento del de scubrimiento, abriendo el a nálisis a lo $\mathrm{s}$ conocimientos sobre una forma de vida sostenible, siempre desde el ent relazamiento de las e ntidades humanas con las entidades no humanas. Asimismo, el mo mento del descubrimiento permite empezar a captar la complejidad de las relaciones observadas, desde la perspectiva de quien está en posibilidad de asumir la función de agencia, es decir, la de poder hacer actuar a otras entida des, mismas que en la "recomposición" de la trama de la vida igualmente pueden ocupar la función de age ncia, con ba se en el rol que pue dan desempeñar.

b) Explorar. Para guia $r$ adecuadamente la exploración, como segundo momento del proceso, es necesario tener en cuenta uno de los principios generales de la AE q ue plantea la idea de que quienes aprenden son "inteligentes por naturaleza", y que cuatro principios específicos orientan la acción de examinar y auscultar: 1) la naturaleza es nuestra maestra; 2 ) la so stenibilidad es una práctica comunitaria; 3 ) el mundo real es el ambiente óptimo para el aprendizaje ecológico, y 4) la vida sostenible se encuentra arraigada en un profu ndo conocimiento del luga r. En efecto, podemos aprender de las sociedades indígenas tradicionales que 
han crecido por siglos siguiendo determinados patrones. Aceptar a la natural eza como maestra ayuda a lo s educadores a centrarse en los principios ecológicos básicos (como la capacidad de soporte o de carga ${ }^{9}$, la Ley del Mínimo de Liebig ${ }^{10}$, los niveles tróficos y energéticos ${ }^{11}$ y la sucesión ecológica ${ }^{12}$ ).

Así, la exploración posibilita comprender la soste nibilidad como práctica colectiva, donde lo más importante es aprender el "arte" de vivir en u n mundo interdependiente. Por lo cual es prioridad mostrar cómo las comunidades actúan en el mu ndo, utilizan recursos y se rela cionan con muchas otras comunidades de las que ellas son un a parte. Los lugares conocidos y am ados profundamente tienen la mejor op ortunidad de ser protegidos y preservados. De e sta manera, ellos podrán alimentar y cuid ar esos lugares para las futuras generaciones. Además, estudiar un lugar en profundidad ayuda a crear un sentido de parentesco o afinidad. También permite a los estudiantes "ver" a través de los ojos de la gente que considera ese lugar como su hogar. Los ayuda a imaginar y contribuir a la solución de los problemas a partir de sus condiciones locales.

c) Enseñar. Una variedad de estrategias de en señanza son particularmente apropiadas para nutrir una vida so stenible. Es importante promover métodos de instrucción pluridisciplinarios que sean adecuados para cada nivel de edad de los alumnos; que vinculen activamente a los e studiantes en el aprendizaje dentro y fuera del salón de clases, que los involucre en proyectos de largo plazo, y pue dan crear una atmósfera de confianza e intercambio de puntos de vista con mucho sentido y reflexió $n$ acerca de t emas ecológicos complejos. Lo principal es el aprendizaje basado en el lugar, ya q ue captura y despierta I a imaginación de los estudiantes y contribuye a una relación de compañerismo y comp romiso cívico. Toda u na serie de herramientas se proporciona a los maestros a fin de que puedan planear e instrumentar un currículum sostenible. Incluye libros, guías de discusión, listados de recursos, lecciones, seminarios de desarrollo profesional, y otro $s$ eventos diseñados por los educadores. Lugar especial ocupa el aprendizaje organizado en torno a proyectos de los estudiantes.

d) Cambiar. La pregunta central es: ¿ Cómo un cambio en la consciencia sobre la sostenibilidad puede repercutir a través de un currículum escolar en la vid a de los estudiantes, en la gobernanza y en la planea ción? En una palab ra: ¿Cómo podemos cambiar los sistemas establecidos? Uno de los principales hallazgos en la experienci a del Centro d e Alfabetización Ecológica es que los cambios, si queremos que sean duraderos, no pueden simplemente ser impuestos por el sistema, como en el caso de la fa milia, la escuela o el distrit o escolar. La experiencia indica que los sistemas por lo regular son resistentes al cambio; y es así como preservan sus identidades y persisten. Pero los sistemas también son e $\mathrm{n}$ parte dinámicos. Asimismo, cada si stema viviente "oca sionalmente" encuentra puntos de i nestabilidad, ante los cuales algunas de sus estructuras se quiebran y emergen nuevas estructuras o nuevas formas de comportamiento. Este fe nómeno es uno de los contrastes de la vida, es la base del aprendizaje, la evolución y la 
innovación. Y así, mientras que los cambios impuestos por un sistema tienen frecuentemente corta vida, es posible crear condiciones que ayuden a determinar si un sistema confrontado con el cambio resiste, colapsa o se abre él mismo a la emergencia de la creatividad y la innovación.

e) Compartir. Para completar los pasos y momentos de I a alfabetización ecológica, con base en las experiencias de Fritjof Capra y colaboradores, es muy i mportante comunicar a otros compañeros las diversas vivencias y resultados obtenidos en su práctica ecológica, ya que favore cen el aco mpañamiento colectivo, ofrecen variedad de casos, tanto exitoso s como problemáticos, para compartir y abren la reflexión a cada uno de los trabajos que las diferentes escuelas están desarrollando. Así, el círculo se cierra y a la vez se a bre, ya que las diferentes presentaciones aportan al conj unto de los participantes las formas y expectativas que orien tan las act ividades de alfabetización. A través de los intercambios van apareciendo con mayor claridad los obstáculos superados, los puntos de soporte para impulsar los cambios, al tie mpo que se acumulan experiencias y se apoyan para llevar adelante sus proyectos. En buena medida se am plía la red d e interesados que e stán llevando a cabo distintas acciones, comprometidos con tratar de avanzar sobre la ba se no de la comp etencia sino de I a cooperación, es de cir, evitando los esquemas de "premios" y concursos para determinar quiénes o c uáles son los mejores trabajos, sino en un encuad re de vinculación entre los estudiantes y sus docentes, que a través de cursos y encuentros planeados, puedan exponer y tambié $\mathrm{n}$ recibir no solo nuevos conocimientos sino además la calidez y comprensión requeridas para sentirse in cluidos e involucrados en los procesos de alfabetización ecológica.

\section{REFLEXIONES FINALES}

- La Alfabetización Ecológica es una concepción de los seres vivos y una práctica ecologista que puede aportar a la diversidad de expresiones y formas de asumir la educación ambiental desde diversas orientaciones y condiciones sociales.

- La AE se presenta como el resultado final y conclusión de un cambio histórico de paradigma, con bases científico-sociales y una experiencia empírica de trabajo con niños y jóvenes para cultivar e impulsar una nueva pedagogía ambiental.

- Como una orientación particular de lo s enfoques no antropocéntricos, su pro pósito central es gen erar cambios institucionales y perso nales a parti $r$ de modificaci ones en lo $s$ procesos de enseñanza-aprendizaje para una vida sostenible.

- El aprendizaje para una vida so stenible es el nú cleo y eje de desarrollo para una verdadera Alfabetización Ecológica. 
- Los principios de la A E se dirig en a promove $r$ un cam bio profundo en la concepción común acerca de la Tierra ylos sistemas vivientes qu e conlleva un a prendizaje basado en el lugar, y que mira al futuro y las siguientes generaciones.

- La nueva pedagogía valora e incluye los conocimientos ecológicos ancestrales, de voz de los pobladores que conservan la sabiduría de sus ante pasados, por lo que combina ciencia y tradiciones en un esquema coherente.

- Asimismo, vincula la escuela con su comunidad, aportando a la conformación de redes heterogéneas a partir de las cuales se organizan y desarrollan los programas ambientales.

- Además, la AE ofrece una perspectiva de la vid a como una totalidad (compleja) en la cual los humanos tenemos una función ineludible en nuestras relaciones de interde pendencia con las entidades no humanas, superando las visiones estrechas y obsoletas para dar paso al optimismo de la acción.

\section{REFERENCIAS}

- Aguilar, M. y Maihol'd, G. (Comps.) (2001). Hacia una cultura ecológica. México: FES.

- Arendt, H. (2002). La vida del espíritu. Buenos Aires: Paidós.

- Bateson, G. (1998). Pasos hacia una ecología de la mente. Buenos Aires: Lohle-Lumen.

- Bertalanffy, L.V. (1974 ). Robots, Hombres y mentes. Madrid: Guadarrama.

- Bocara, N. (2004 ). Modeling complex Systems. Nueva Yo rk: Springer.

- Capra, F. (1999). La trama de la vida. Barcelona: Anagrama.

- (2003). Las conexiones ocultas. Madrid: Anagrama.

- Capra, F. y Luisi, P.L. (201 4). The Systems View of Life: A Unifying Vision. Cambridge: Cambridge University Press.

- Carabias, J., Meave, J.A ., Valverde, T. y Can o-Santana, Z. (2009). Ecología y medio ambiente en el siglo XXI. México: Oearson-Prentice Hall.

- Caride, J.A. y Metra, P.A. (2001). Educación ambiental y desarrollo humano. Barcelona: Editorial Ariel.

- Damian, R. y Monteleon e; A. (2002). Temas ambientales en el aula. Buenos Aires: Paidós.

- Deléage, J.P. (1993). Historia de la Ecología, una ciencia del hombre y de la naturaleza. España: Icaria.

- Diamond, J. (2007). Colapso. Madrid: Ed. El Bolsillo.

- Eve, R, Horsfall, S. y Lee, M.E. (1997). Chaos, complexity and sociology: Myths, models and theories. Thousand Oaks: Sage.

- García, M. (2005). Ecología profunda y educación. Memoria para obtener el grado de doctor. Universidad Complutense de Madrid, Madrid.

- García, R. (2005). Una polémica que conserva actualidad. El Arca Digital, 56. Re cuperado de 
http//www.elarcadigital.com.ar/elarca/numerosanteriores/ARCA56/ PDF 56/09CAOS56.PDF

- Guattari, F. (1996). Caosmosis. Buenos Aires: Manantial.

- Guiddens, A. (1998). Modernidad e identidad del yo. Barcelona: Península.

- Gutiérrez, J. (1995). La educación ambiental: Fundamentos teóricos, propuestas de transversalidad y orientaciones extracurriculares. Madrid: La Muralla.

- Harvey, D.L. y Reed, M. (1997). Social sciences as the study of complex Systems. En Ki el, L.D. y Elliot, E. (Comps.), Chaos theory in the social sciences. Foundations and applications (pp. 295-323). Ann Arbor: The University of Michigan Press.

- Hayles, K. (2003 ). La evolución del caos: el orden dentro del desorden en las ciencias contemporáneas. Barcelona: Gedisa.

- Issa, J. (2 003). El giro p ragmático en la Ética Ambiental. En Kwiatkowska, T. e ls sa, J. (Comps .), Los caminos de la Ética Ambiental (pp. 85-92). II. México: Plaza y Valdés.

- Jacob, F. (1994). La lógica de lo viviente. Barcelona: Laia.

- Jantsch, E. (1980). The Self-Organizing Universe. Nueva York: Pergamon.

- Jonassen, D. y La nd, S. (Co mps.) (2000). Theoretical Foundations of Learning Environments, Mahwah, Nueva Je rsey: Lawrence Erlbaum.

- Kierkegaard, S.(1965 ). El concepto de angustia (trad.d e Demetrio Rivero). Madrid: Guadarrama.

- Lovelock, J. y Margulis, L. (1974). Biological Modul ation of the Earth's Atmosphere. Icarus, 21(4), 471-489.

- Martínez Mekler, G. (1993). Di námica y estru ctura de si stemas complejos. En García-Colín, L., Ramos Gómez, F. y Rechtman, R. (Coords.), Temas selectos de física estadística (pp. 141-227). México: El Colegio Nacional.

- Martínez Mekler, G. y Goch o, G. (1998). Al bord e del milenio: caos, crisis y complejidad. En De la Peña, L. (Comp.), Ciencias de la materia. Génesis y evolución de sus conceptos fundamentales. México: Siglo XXI Editores.

- Maturana, H. y Varela, F . (2000). El Árbol del Conocimiento. Buenos Aires: Debate.

- (2001). Auotopoiesis: The organization of the Living. Santiago de Chile: Editorial Universitaria.

- Merchant, C. (1992). Radical Ecology: The Search for a Liable World. New York: Routledge.

- Monod, J. (1998). El azar y la necesidad. Barcelona: Tusquets.

- Naess, A. (1969). Scepticism. New York: Humanities Press.

- $\quad$ (1988). Deep Ecology and Ultimat e Premises. Ecologist, 18(4-5), 128-131.

- $\quad$ (1995). Self Realization. An Ecologi cal Approach to Being in the world. En Sessions, Deep Ecology for the 21st Century. p. 224.

- $\quad$ (2002). Life's Philosophy: Reason \& Feeling in a Deeper World. Athens, Georgia: University of Georgia Press.

- Passmore, J. (1978). La responsabilidad del hombre frente a la naturaleza (trad. de Álvaro Delgado). Madrid: Alianza.

- Penfield, W. (1995). The Mysteries of the Mind. Nueva Jersey: Princeton University Press. 
- Pomier, P. (2002). Determinismo biológico: el desafío de la Alfabetización Ecológica en I a concepción de Fritjof Capra. Tópicos en Educación Ambiental, 4(11), 7-18.

- Prigogine, I. (2008). Las leyes del caos. Barcelona: Drakontos.

- Prigogine, I. y Stengers, I. (2004 ). Order out of Chaos. Nueva York: Bantam.

- Reynoso, C. (2009). Modelos o metáforas. Crítica del paradigma de la complejidad de Edgar Morin. Buenos Aires: Editorial SB.

- Rozsak, Theodore (2003). The voice of the earth. Nueva York: Bantam Press.

- Sessions, G. (Ed.) (199 5). Deep Ecology for the 21st Century: Readings on the Philosophy and Practice of the New Environmentalism. 1 $^{\text {a }}$. Ed. Boston, MA: Shambhala.

- Sureda, J. y Colom, A.J. (2003). Pedagogía ambiental. Barcelona: CEAC.

- Schrödinger, E. (2012). ¿Qué es la vida? México: Tusquets Editores.

- Wagensberg, J. (2 001). La necesidad del azar. Barcelona: Gedisa.

- Wenger, E. (2001). Comunidades de práctica, aprendizaje, significado e identidad. Barcelona: Paidós.

- West, T. (2002). Ecología y educación. Elementos para el análisis de la dimensión ambiental en el currículum escolar. México: Universidad

Nacional Autónoma de México.

- Wolf, F.A. (2013). Bucles temporales y pliegues espaciales. Barcelona: Ediciones Obelisco.

1. Profesor-Investigador de la Universidad Autónoma del Estado de México, adscrito a la Facultad de Artes. arandasjm@gmail.com

2. Sistemas complejos: totalidades funcionales caracterizadas por sus componentes interconectados e interdependientes, diversos, adaptativos, dependientes del camino, y emergentes (no lineales, y multi-nivel).

3. En términos de Kierkeg aard. Ver especialmente: Kierkegaard (1965).

4. Corriente de la Psicolog ía moderna, surgida en Alemania a principios del siglo XX. Sostiene, con base en i nvestigación empírica, que la 'buena forma' o la más sencilla es la que domina la captación de los contextos, otorgándole a la percepción de la configuración el papel central en la apropiación del entorno.

5. Comunidades ecológicas o bióticas: conjuntos de poblaciones de diferentes especies que conviven en un sitio d onde pueden interactuar, al menos potencialmente, de diversas formas.

6. Para profundizar en el te ma y sus co mplejidades, ver Fred Alan Wolf (2013).

7. En lo relativo a la escala social.

8. Desarrollo sostenible es el término que se le da al equilibrio del manejo del planeta en tres ámbitos: ambiental, social y económico. Teniendo en cuenta que ningún recurso renovable deberá utilizarse a un ritmo superior al de su generación, ningún contaminante deberá producirse a un ritmo superior al que pueda ser reciclado, neutralizado o absorbi do por el me dio ambiente, ningún recurso no renov able deberá aprovecharse a mayo $r$ 
velocidad de la nece saria para sustituirlo $p$ or un recurso renovable utilizado de manera sostenible. Mientras que el desarrollo sustentable alude al manejo de los recursos naturales, humanos, sociales, económicos y tecnológicos, con el fin de alcanzar una mayor calidad de vida para la po blación y, al mismo tiempo, velar porque los patrones de consumo actual no afecten el bienestar de las generaciones futuras.

9. Tamaño o den sidad máxima de u na población que pu ede soportar un ambiente dado. Es la d ensidad poblacional a la cual la tasa de nacimientos es igual a la tasa de muertes y, por lo tanto, el crecimiento poblacional es de cero.

10. Principio de la Ecofisiología que establece que el rendimiento de un organismo depende del recurso que presenta menor disponibilidad en un hábitat, es de cir, del qu e más limita su desempeño.

11. Posición que guardan los o rganismos o sus actividades alimenticias en la cadena alimentaria. Los organismos del primer nivel trófico son lo s productores primarios, los del segundo nivel son los con sumidores primarios yl os del tercero son los consumidores secundarios.

12. Proceso de cambi o direccional (no cí clico) en un a comunidad ecológica a través del tiempo. Su inicio gen eralmente está asociado a un disturbio. Pueden darse: sucesión primaria, donde el cambio se da a pa rtir de un disturbio que elimina todos los elementos bióticos; o su cesión secundaria, donde el cambio se genera a partir de un disturbio que permite la permanencia de una fracción de elementos bióticos.

Para citar este artículo: Aranda S ánchez, J.M. (2015). La Alfabetización Ecológica como nue va pedagogía para la comprensión de los seres viviente. Revista Luna Azul, 41, 365384. Recuperado http://lunazul.ucaldas.edu.co/index.php?option=content\&tas k=view\&id=1071 